TIFR/TH/09-05

\title{
Relativistic diffusion and heavy-ion collisions
}

\author{
Rajeev S. Bhalerao* and Sourendu Gupta ${ }^{\dagger}$ \\ Department of Theoretical Physics, \\ Tata Institute of Fundamental Research, \\ Homi Bhabha Road, Mumbai 400005, India.
}

\begin{abstract}
We study first- and second-order theories of relativistic diffusion coupled to hydrodynamics under the approximation, valid at mid-rapidity in the RHIC and LHC, that conserved number densities are much smaller than the entropy density. We identify experimentally accessible quantities of interest, and show that the first- and second-order theories may lead to radically different evolutions of these quantities. In the first-order theory the memory of the initial state is almost completely washed out, whereas in the second order theory it is possible that freezeout occurs at a time when transient dynamics is still on, and the memory of the initial state remains. There are observational consequences which we touch upon. In the first-order theory, and for initial conditions when the second-order theory mimics the first-order, one may be able to put a bound on the diffusion constant.
\end{abstract}

Event-to-event fluctuations of conserved quantum numbers in heavy-ion collisions have been espoused as signals of the underlying thermodynamics [1]. Quantities which have been considered interesting include the baryon number, $B$, the electric charge, $Q$, and even the strangeness, $S$, which is conserved in strong interactions. The proton number, $N_{p}$, has been suggested as a proxy for $B$ [2]. Of interest are the net conserved quantum number and its distribution over the ensemble of events at the collider.

In every observation of a collision event, one can find the density of $B, Q$ or $S$ as a function of rapidity. In the usual measurement of fluctuations one usually sums such a density over all rapidity within the observational window: this corresponds to taking the Fourier coefficient $k=0$ of the density profile. However, other Fourier coefficients can be easily constructed. In this paper we point out that hydrodynamic evolution of the Fourier coefficients of conserved number densities are interesting in their own right, since they may contain interesting signals not only of the initial conditions but also of hydrodynamic evolution.

To this end we investigate the coupled evolution of the number densities and the usual hydrodynamic quantities, i.e., the stress tensor, $T_{\mu \nu}$, expressed as usual in terms of the field of flow velocity, $u_{\mu}$, and the energy density, $\epsilon$, or the entropy density, $s$, and the dissipative parts. In heavy-ion collisions at the RHIC net conserved number densities are small compared to the entropy density. This is usually expressed as the observation that the ratio of the appropriate chemical potential, $\mu$, and the temperature, $T$, i.e., $\mu / T$, is small [3]. This ratio is expected to become even smaller at the forthcoming LHC experiment. As a result, the finite $\mu$ corrections to the pressure, $p$, the speed of sound, $c_{s}$, and to $\epsilon$ and $s$ are expected to be small. The combination of these quantities along with the number densities obey a set of coupled equations. The smallness of $\mu / T$ implies that the equations can be linearized in $\mu / T$, and an accurate picture of the hydrodynamics is obtained by considering the diffusion equation coupled to the hydrodynamic flow while neglecting the effect of the number densities on the flow. We note that this approximation is made here for convenience. It allows us to understand the essential physics of the situation without much of a sacrifice in accuracy. If higher accuracy is needed, one can easily investigate the fully coupled formalism [4].

Previous work had investigated heavy quark diffusion in the heavy-ion induced fireball [5]. The energy loss of the heavy-quark was estimated using weak-coupling theory [6], the Boltzmann equation [7], AdS/CFT techniques [8], and using Langevin dynamics [9]. A diffusive theory has also been invoked to describe stopping and early time entropy production [10]. Our motivation, and therefore, the theory we develop, is quite different.

In this paper we consider only longitudinal background fluid flow, i.e., flow in which the $z$-axis is defined by the direction of the incoming nuclei, and the dependence of all quantities on the orthogonal $x$ and $y$ coordinates is neglected. This first approximation is expected to be valid in nearly head-on (small impact parameter) collisions of nuclei for times shorter than the sound travel time across the fireball, i.e., for $\tau \leq R / c_{s}$, where $R$ is the radius of the colliding nuclei. We work with curvilinear coordinates, i.e., the space-time rapidity, $\eta=(1 / 2) \log (t+z) /(t-z)$, and the epoch, $\tau=\sqrt{t^{2}-z^{2}}$. The covariant derivatives, $d_{\mu}$ then include Christoffel symbols. We define the local timelike derivative, $D=u^{\mu} d_{\mu}$. This allows us to define a vector $v_{\mu}=\left(D u_{\mu}\right) / S$ which is a spacelike unit vector orthogonal to $u_{\mu}$, where $S^{2}=-\left(D u_{\mu}\right)\left(D u^{\mu}\right)$. In terms of this new vector we define the spacelike derivative $\widetilde{D}=v^{\mu} d_{\mu}$. The spacelike projector is $\Delta_{\mu \nu}=g_{\mu \nu}-u_{\mu} u_{\nu}$. A scalar which appears often is $\Theta=d_{\mu} u^{\mu}$. For longitudinal flow we can parametrize the fluid velocity as $u_{\tau}=\cosh y$ and $\tau u_{\eta}=\sinh y$ with the other two components vanishing. Boost invariant flow

*Electronic address: bhalerao@tifr.res.in

$\dagger$ Electronic address: sgupta@tifr.res.in 
corresponds to taking $y=0$ everywhere. This is not the same as the fluid being at rest; the choice of the particular curvilinear coordinates that we use makes this simple parametrization possible. Then one has $\Theta=1 / \tau$ and $S=0$ (note that $v_{\mu}$ remains finite). The derivative $D=\partial / \partial \tau$ and $\tau \widetilde{D}=\partial / \partial \eta$. In all this we follow the notation of [11], to which we refer readers for further details.

We examine the kinematics of the number current, $n_{\mu}$. One finds the number density as usual, $n=n_{\mu} u^{\mu}$. Then one can write $n_{\mu}=n u_{\mu}+\nu v_{\mu}$, where $\nu v_{\mu}$ is the dissipative part of the number current. Clearly, one has $\nu=-v_{\mu} n^{\mu}$. When there is more than one conserved particle number, one can write an analogous decomposition for each conserved quantity, with different $n$ and $\nu$ for each. For each conserved number, the equation of continuity is

$$
0=d_{\mu} n^{\mu}=D n+n \Theta+\widetilde{D} \nu+S \nu
$$

We will assume in this paper that the background fluid undergoes boost invariant longitudinal flow. However, as a result of the assumption that $\mu / T \ll 1$, there is no contradiction in assuming that $n_{\mu}$ is not boost invariant while the background flow is. In fact, the physics of diffusion, which is the central object of interest here, would be trivially absent if $n$ and $\nu$ were both independent of $\eta$.

In ideal fluids there is no dissipative part to any hydrodynamic quantity, so $\nu=0$. The hydrodynamics then lies entirely in the continuity equation and its self-similar solution

$$
\frac{d n(\tau, \eta)}{d \tau}=-\frac{n(\tau, \eta)}{\tau}, \quad \text { so that } \quad \frac{n(\tau, \eta)}{n\left(\tau_{0}, \eta\right)}=\frac{\tau_{0}}{\tau} .
$$

Any initial number density profile is then propagated in time unchanged in shape but attenuated as $1 / \tau$ due to the geometry of longitudinal flow. We call this phenomenon Bjorken attenuation.

Following [11] we define the Fourier transform and the power spectrum of the density, respectively, as

$$
n(\tau, k)=\frac{1}{\sqrt{2 \pi}} \int d \eta n(\tau, \eta) \exp [-i k \eta], \quad \text { and } \quad P(\tau, k)=|n(\tau, k)|^{2} .
$$

Note that the measure used here is $d \eta$, whereas the invariant volume measure contains $\tau d \eta$. Hence, the Fourier coefficient $n(\tau, k=0)$ is not the conserved charge, but $\tau n(\tau, k=0)$ is conserved. For an ideal fluid all Fourier coefficients of the number density profile also undergo Bjorken attenuation, which, as we see, is a purely geometric phenomenon for boost invariant flows.

For non-ideal fluids the first order constitutive equation for diffusion is Fick's Law. In the local rest frame of the fluid this takes the form

$$
0=n^{i}+\mathcal{D} \partial^{i} n
$$

where $\mathcal{D}$ is the diffusion coefficient. In a general frame one can recast this in the form

$$
0=\Delta^{\mu \nu} n_{\nu}-\mathcal{D} \Delta^{\mu \nu} d_{\nu} n=\nu v^{\mu}-\mathcal{D}\left[d^{\mu} n-u^{\mu} D n\right]
$$

using the projector orthogonal to $u$. When the last form on the right is contracted with $u^{\mu}$, one finds that it vanishes trivially. A contraction with $v^{\mu}$ relates the dissipative part with the spatial derivative of the number density-

$$
0=-\nu-\mathcal{D} \widetilde{D} n
$$

Substituting this form of Fick's law into the continuity equation and thereby eliminating $\nu$, one finds the relativistic version of the diffusion equation in a fluid undergoing longitudinal expansion-

$$
0=D n-\widetilde{D} \mathcal{D} \widetilde{D} n-\mathcal{D} S \widetilde{D} n+n \Theta .
$$

Note that the last two terms are directly related to the hydrodynamic flow. Since the equation is linear, it can be solved by Fourier transforming in the spatial variable and considering the evolution of each Fourier mode separately. For later convenience we give the name Fick diffusion to phenomena that arise from this equation.

The relative importance of flow and continuity versus diffusion can be quantified through the dimensionless quantity which compares the 2 nd and the last terms in eq. (7) -

$$
\mathcal{W}=\frac{\lambda^{2}}{\tau \mathcal{D}}=\frac{\tau}{\mathcal{D}} \sinh ^{2} \Delta \eta
$$

where $\lambda=\tau \sinh \Delta \eta$ is an intrinsic length scale in the density profile, corresponding to a scale $\Delta \eta$ in rapidity. When $\mathcal{W} \ll 1$ then diffusive effects dominate over flow; when it is much greater than unity, flow dominates. Which behaviour 
dominates depends also on how $\mathcal{D}$ changes with time. In the high temperature phase of the plasma, where there is essentially only one scale, $T$, one has $\mathcal{D} \propto 1 / T$ (in an AdS/CFT computation one typically obtains $\mathcal{D} T=1 / 2 \pi$, although much smaller and larger results can also be obtained by tuning parameters [12]). However, at sufficiently small $T$ (close to $T_{c}$, for example) it is possible that $\mathcal{D}$ is controlled by a hadronic length scale, and may change only marginally with $T$. In the high temperature phase, if the background flow is boost invariant and longitudinal, then $T \propto 1 / \tau^{\left(1+c_{s}^{2}\right) / 4}$. In this case $\mathcal{W}$ increases with $\tau$, leading to a decreased importance of the diffusive term. At lower temperature as well, since $\mathcal{D}$ varies little, $\mathcal{W}$ increases and the flow terms become more important. If we are concerned with a $\Delta \eta$ range such that diffusion dominates at initial times, then after a time $\tau_{f l}=\mathcal{D} / \sinh ^{2} \Delta \eta$, flow dominates over diffusion. If at times less than $\tau_{f l}$ diffusion manages to destroy structures in the number density profile, then the memory of the initial state can be lost.

This can be seen in a simple model of a fluid where all transport coefficients are constant and independent of parameters such as $\mu$ and $T$. Then, for any longitudinal background flow of such a fluid the diffusion equation (7) becomes

$$
0=\frac{\partial n}{\partial \tau}+\frac{n}{\tau}-\frac{\mathcal{D}}{\tau^{2}} \frac{\partial^{2} n}{\partial \eta^{2}}
$$

after linearizing in $\mu / T$. The Fourier coefficients of $n$ obey the equation

$$
0=\frac{\partial n(\tau, k)}{\partial \tau}+\frac{n(\tau, k)}{\tau}+\frac{\mathcal{D} k^{2}}{\tau^{2}} n(\tau, k) .
$$

The solution can be obtained by quadrature-

$$
n(\tau, k)=n\left(\tau_{0}, k\right)\left(\frac{\tau_{0}}{\tau}\right) \exp \left[-\frac{\mathcal{D} k^{2}}{\tau_{0}}\left(1-\frac{\tau_{0}}{\tau}\right)\right] .
$$

The constant mode $(k=0)$ exhibits Bjorken attenuation, as all Fourier components eventually do, consistent with the analysis of $\mathcal{W}$. Behaviour typical of first-order diffusion is the relative suppression of modes with larger $k$, i.e., the monotonic smoothing of any initial density profile.

It is possible for experiments to give an upper bound for $\mathcal{D}$. If one sees no structure in the number density profile for rapidity separations up to $\Delta \eta$ then one can conclude either that structures on such scales were not present in the initial state, or that they were present and were wiped out by diffusion. The time available for diffusion to act is bounded by the freezeout epoch, $\tau_{f}$. Hence, one gets the limit $\mathcal{D} \leq \tau_{f} \sinh ^{2} \Delta \eta$. One should be careful about this upper limit for $\mathcal{D}$, if $\Delta \eta$ is very small, i.e., if one observes fluctuations in the number density profile at all scales. There is a sum rule between $\mathcal{D}$ and the relaxation time for diffusive processes, $\tau_{R}[13,14]: \mathcal{D}=c_{s}^{2} \tau_{R}$. When freezout occurs away from a critical point, a vanishing $\mathcal{D}$ implies a vanishing $\tau_{R}$, whereas we expect that $\tau_{R}$ is not zero. Hence, if we see fluctuations of number densities at all scales $\Delta \eta$, then it is likely that the first order theory breaks down. To find what could happen then, we next investigate the second order theory.

At second order, Fick's law, eq. (4), can be replaced by the form [13, 14]

$$
0=\left(1+\tau_{R} \partial_{t}\right) n^{i}+\mathcal{D} \partial^{i} n
$$

where $\tau_{R}$ is a relaxation time for number changing processes. This equation is written in the rest frame of the fluid. One uses the projector orthogonal to $u$ to write this covariantly. The covariant equation is

$$
0=\nu v^{\mu}+\tau_{R} \Delta^{\mu \nu} D n_{\nu}-\mathcal{D}\left(d^{\mu} n-u^{\mu} D n\right) .
$$

Projecting parallel to $u$ gives 0 identically. Projecting parallel to $v$ and using the definition $D u_{\mu}=S v_{\mu}$ and the identity $v_{\mu} D v^{\mu}=0$ gives

$$
\nu+\tau_{R} D \nu=-\mathcal{D} \widetilde{D} n-S \tau_{R} n
$$

This replaces the constraint (eq. 6) for $\nu$ obtained in the first order formalism. Kelly makes the identification $\mathcal{D} / \tau_{R}=c_{s}^{2}$, and we retain this intuition in this work. The quantity $\tau_{R}$ is currently unknown; it could be of the order of typical QCD scales, i.e., $1 \mathrm{fm}$, or it could be significantly smaller in AdS/QCD scenarios, for example, $0.1 \mathrm{fm}$.

In the background of boost-invariant longitudinal flows, eqs. (1) and (14) reduce to

$$
0=\frac{\partial n}{\partial \tau}+\frac{n}{\tau}+\frac{1}{\tau} \frac{\partial \nu}{\partial \eta}, \quad \text { and } \quad 0=\tau_{R} \frac{\partial \nu}{\partial \tau}+\nu+\frac{\mathcal{D}}{\tau} \frac{\partial n}{\partial \eta}
$$


This is the form that Kelly's second order diffusion equation [13] takes in a boost-invariant geometry. In general one expects the diffusion constant $\mathcal{D}$ and the relaxation time $\tau_{R}$ to depend on the temperature, $T$. Since the time-evolution of $T$ is obtained by solving the remaining hydrodynamic equations, one gets an explicit time dependence of $\mathcal{D}$ and $\tau_{R}$, and hence one can solve the equations by Fourier transformation. The evolution equations for the Fourier modes can be written in the form

$$
\frac{\partial}{\partial \tau}\left(\begin{array}{c}
n(\tau, k) \\
\nu(\tau, k)
\end{array}\right)=-\left(\begin{array}{cc}
1 / \tau & i k / \tau \\
i c_{s}^{2} k / \tau & 1 / \tau_{R}
\end{array}\right)\left(\begin{array}{c}
n(\tau, k) \\
\nu(\tau, k)
\end{array}\right)
$$

Since $c_{s}^{2}$ varies within a bounded region, $c_{s}^{2} / \tau$ falls with $\tau$, and at late times $\nu$ decays as $\exp \left(-\tau / \tau_{R}\right)$, and $n$ decays as $1 / \tau$, thus reducing to Bjorken attenuation at late times. The $k=0$ mode exhibits Bjorken attenuation at all times.

For more detailed analysis it is useful to change variables to $\vartheta=\log \left(\tau / \tau_{R}\right)$. The equations then become

$$
\frac{\partial}{\partial \vartheta}\left(\begin{array}{c}
n(\vartheta, k) \\
\nu(\vartheta, k)
\end{array}\right)=-M\left(\begin{array}{c}
n(\vartheta, k) \\
\nu(\vartheta, k)
\end{array}\right), \quad M=\left(\begin{array}{cc}
1 & i k \\
i c_{s}^{2} k & \mathrm{e}^{\vartheta}
\end{array}\right)
$$

Note three regions of Fourier modes-

1. When $k$ is sufficiently smaller than unity, the off-diagonal terms may be neglected to a good approximation, and the problem decouples. The equation for $n(\vartheta, k)$ is then similar to the ideal case. We do not discuss the $k=0$ mode in the following.

2. When $k$ is sufficiently larger than $\exp (\vartheta)$, the problem simplifies again. However, the hydrodynamic equations are an approximation to microscopic physics, and are valid for long wavelength spatial fluctuations and for long-time phenomena. The time scales are $\exp \vartheta>1$, and the limit of $k \rightarrow \infty$ must be discarded, although the equations simplify.

3. In the remaining region the equations are fully coupled and a more detailed analysis is called for. This follows.

Since $M$ depends explicitly on $\vartheta$, a complete solution to the differential equations cannot be obtained by just diagonalizing it. First note that when $k \neq 0$, then for either $\vartheta \neq 0$ or $c_{s}^{2} \neq 1$, one has $\left[M, M^{\dagger}\right] \neq 0$, i.e., $M$ is non-normal. As a result, $M$ is not diagonalized by a unitary transformation, and the eigenvectors are not orthogonal. For non-normal matrices the concept of pseudospectra can yield powerful results [15]. In this paper, however, we present a standard spectral analysis, since very detailed information can be obtained by this route.

Since the trace and determinant of $M$ are real, the eigenvalues are either both real or both complex,

$$
\lambda_{ \pm}=\frac{1}{2}\left(1+\mathrm{e}^{\vartheta}\right) \pm \frac{1}{2} \sqrt{\left(\mathrm{e}^{\vartheta}-1\right)^{2}-4 c_{s}^{2} k^{2}}
$$

For a given $\vartheta$, the eigenvalues are real for $|k| \leq\left(\mathrm{e}^{\vartheta}-1\right) /\left(2 c_{s}\right)$. When they are real, then they are both positive since the first term of the expression in eq. (18) is greater than $\mathrm{e}^{\vartheta} / 2$ and the second term is less than $\mathrm{e}^{\vartheta} / 2$. When both are complex, then the real parts are positive, since the trace is so. This implies that $|n|^{2}$ and $|\nu|^{2}$ both must decay at long times. However, there may be transient growth.

One way to show that transient growth may occur in general is to write down the evolution equation for the power spectrum

$$
P(\vartheta, k)=|n(\vartheta, k)|^{2}=\mathbf{x}^{\dagger} A \mathbf{x} \quad \text { where } \quad \mathbf{x}=\left(\begin{array}{l}
n \\
\nu
\end{array}\right) \quad \text { and } \quad A=\left(\begin{array}{ll}
1 & 0 \\
0 & 0
\end{array}\right)
$$

The evolution equation is

$$
\frac{d P(\vartheta, k)}{d \vartheta}=\frac{d \mathbf{x}^{\dagger}}{d \vartheta} A \mathbf{x}+\mathbf{x}^{\dagger} A \frac{d \mathbf{x}}{d \vartheta}=-\mathbf{x}^{\dagger} \mathcal{M} \mathbf{x}, \quad \text { where } \quad \mathcal{M}=\left(\begin{array}{cc}
2 & i k \\
-i k & 0
\end{array}\right)
$$

independent of $c_{s}^{2}$ and $\vartheta$. The quantity $-\mathbf{x}^{\dagger} \mathcal{M} \mathbf{x} /|x|^{2}$ is called the numerical range of the matrix $-\mathcal{M}$, and is bounded by the eigenvalues of $-\mathcal{M}$, since the matrix is Hermitean. Since the determinant is negative, one of the eigenvalues is positive and the other negative. As a result, the numerical range has indefinite sign, and amplification of the power spectrum is possible. The vectors $\mathbf{x}$ may be parametrized as $\mathbf{x}^{\dagger}=x\left(\sin \theta \mathrm{e}^{-i \phi}, \cos \theta\right)$, where $\theta$ and $\phi$ range over the sphere. Then one has

$$
-\mathbf{x}^{\dagger} \mathcal{M} \mathbf{x}=-2|x|^{2} \sin \theta(\sin \theta+k \cos \theta \sin \phi)
$$




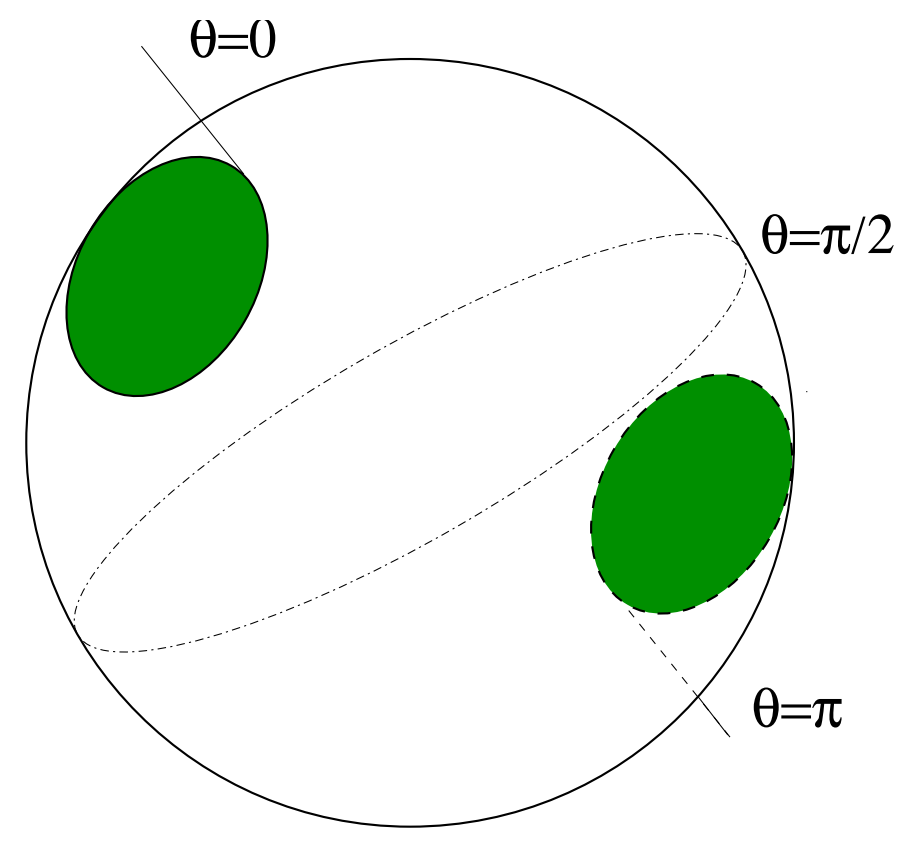

FIG. 1: The parts of the sphere in which the power spectrum is amplified are indicated by the green patches. They are symmetrically placed at the poles and are bounded in the region $0 \leq \theta \leq \tan ^{-1} k$ and symmetrically in $\pi+\tan ^{-1}(-k) \leq \theta \leq \pi$.

At the equator, $\theta=\pi / 2$, this quantity is negative. There are zeroes at the poles, $\theta=0$ and $\pi$. Other zeroes occur along the curves $\tan \theta=-k \sin \phi$. This equation describes two closed curves on the sphere each of which passes through one of the poles (see Figure 1). In the area of the sphere inside these curves (not containing the equator) the function is positive. Amplification of $P(\vartheta, k)$ can take place whenever $\mathbf{x}$ passes through the non-empty region where $\mathbf{x}^{\dagger} \mathcal{M} \mathbf{x}<0$.

Since initial conditions change from event to event, the probability of transient growth is measured by the fractional area of the sphere where transient growth may occur. The larger the value of $k$, the closer does the zero curve come to the equator, and hence the larger the probability of transient growth becomes. However, large $k$ corresponds to smaller $\Delta \eta$, and hence to short range structures in rapidity. Since such structures are efficiently erased in Fick diffusion, their observation in a significant fraction of events would serve as a signal of Kelly diffusion.
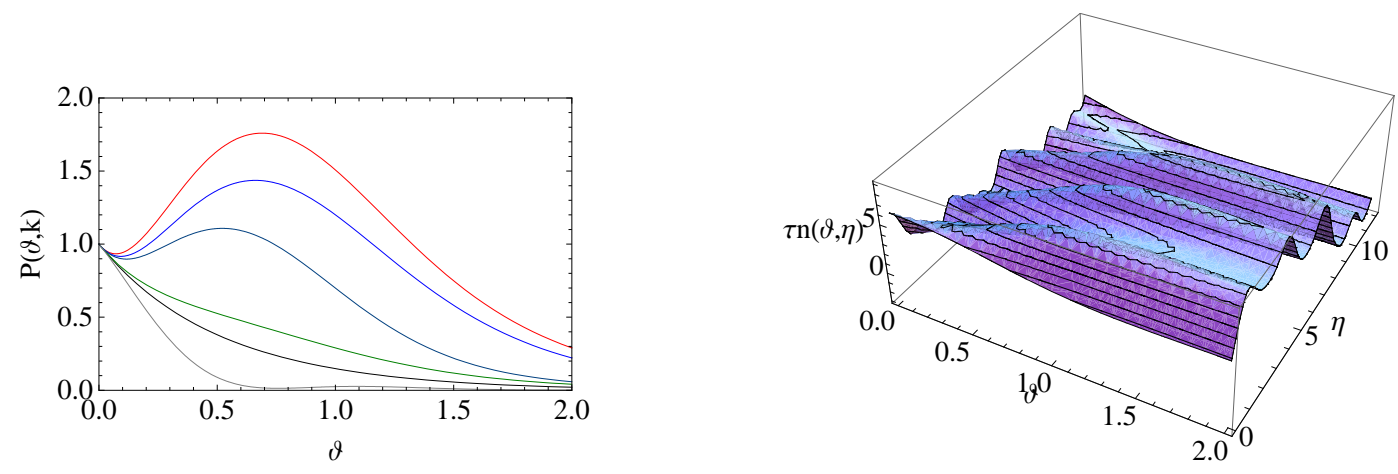

FIG. 2: The evolution of the power spectrum, $P(\vartheta, k)$, and the number profile, $\tau n(\vartheta, \eta)$, for initial conditions containing only a discrete set of non-vanishing modes $k=1 / 8$ (black), 1/4 (red), 1/2 (green), 1 (blue), 2 (dark blue) and 4 (gray), which are taken with a flat power spectrum at $t=0$. We have chosen $c_{s}^{2}=1 / 3$ for this example. The initial conditions on the corresponding $\nu(0, k)$ are assigned at random. The evolution of the power spectrum is shown on the left and of the profile on the right. In contrast to the intuition from the Fick theory, the profile may steepen at intermediate times.

In Figure 2 we show an example of the evolution of number density profiles using eq. (17), starting from random initial conditions. In terms of Fourier modes it is clear that transient growth may occur for any $k$. The typical time scale of this growth is $\vartheta \approx 1$, i.e., $\tau \approx e \tau_{R}$. However, even at $\vartheta \approx 2$, i.e., $\tau \approx e^{2} \tau_{R}$ the evolution does not begin to 

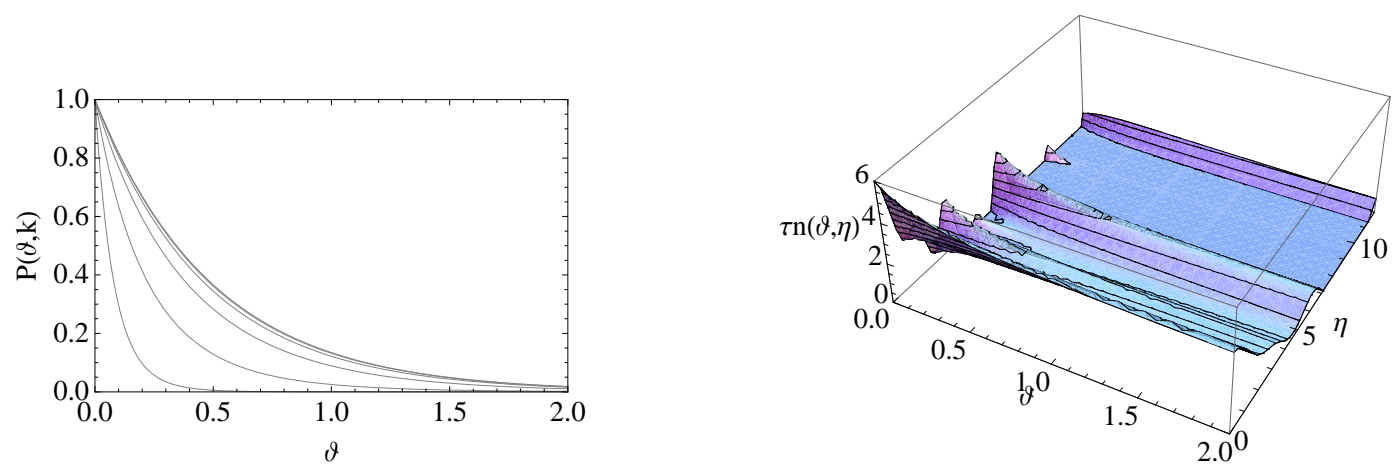

FIG. 3: Same as Figure 2, but using the first order diffusion equation. The evolution of the power spectrum is governed by eq. (11).

resemble Bjorken attenuation. If $\tau_{R}$ is in the range of $1 / 2$ to $2 \mathrm{fm}$, then the evolution of number densities may be dominated by transients. Such long time scales are good news, because they allow one to extract information on the early stages of the fireball.

Figure 3 shows the evolution in the Fick theory of $P(\vartheta, k)$ and $\tau n(\vartheta, k)$ starting from the same initial conditions as above. The difference between first order (Fick) and second order (Kelly) theories of diffusion seems to be fairly fundamental. The main intuitive understanding of diffusion from the Fick theory is the following: if initial conditions set up sharp density gradients, then diffusion always smooths these out on all scales, monotonically, i.e., as time evolves the gradients get smoother and smoother. The Kelly theory can violate this intuition. Depending on initial conditions, gradients may be transiently amplified, at different scales at different times. The asymptotic final state is the same in both theories. However, since freezeout may not occur at asymptotically late times, the number density profiles at freezeout could be different. Some feel of the differences between the theories can be obtained by comparing Figures 2 and 3. Note, in particular, that the solution of Kelly's equation contains structure in the number density profile at scales of $\Delta \eta \simeq 1$ even at times $\tau \simeq 7.5 \tau_{R}$. In contrast, for the solution of Fick's equation, the scales of $\Delta \eta$ on which structures are seen are much larger.

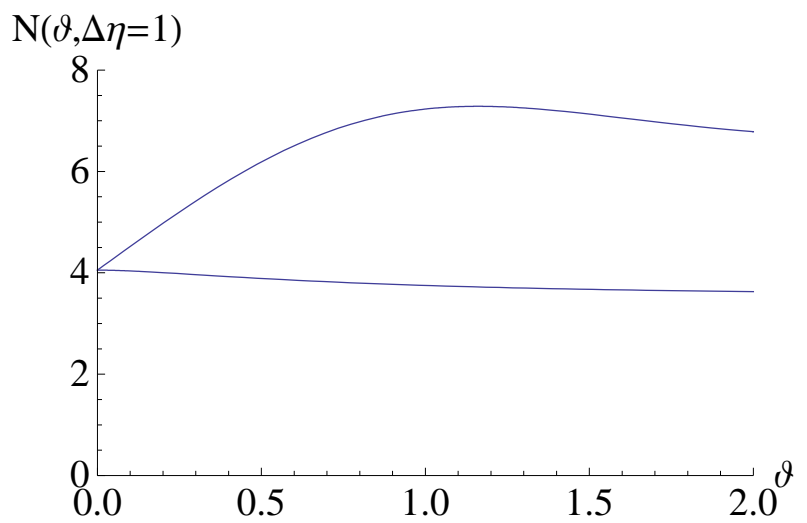

FIG. 4: The total conserved charge, $N$, within a window $\Delta \eta=1$ (between $\eta=0$ and $\eta=1$ ) for the initial conditions used in Figures 2 and 3. The upper line is for the Kelly theory and the lower one for the Fick theory.

In Figure 4 we show the conserved charge, $N(\vartheta, \Delta \eta=1)$, in a rapidity window $\Delta \eta=1$ (between $\eta=0$ and $\eta=1$ ) as a function of $\vartheta$. In both theories there is a tendency for the charge to become independent of $\vartheta$ at late times. This happens because diffusion must eventually wipe out all spatial structure in $n(\vartheta, \eta)$, and once the profile has flattened, the number density is locally conserved. The differences between the two theories are due precisely to the transients that we have studied. In the Fick theory transient lifetimes are extremely small even for $k<1$, hence the asymptotic behaviour sets in very early. This can also be seen from the profiles in Figure 3. In the Kelly theory, on the other hand, transients could be long lasting (depending on initial conditions). This is seen in the evolution of the density profiles in Figure 2, as well as of $N(\vartheta, \Delta \eta=1)$ (Figure 4). 

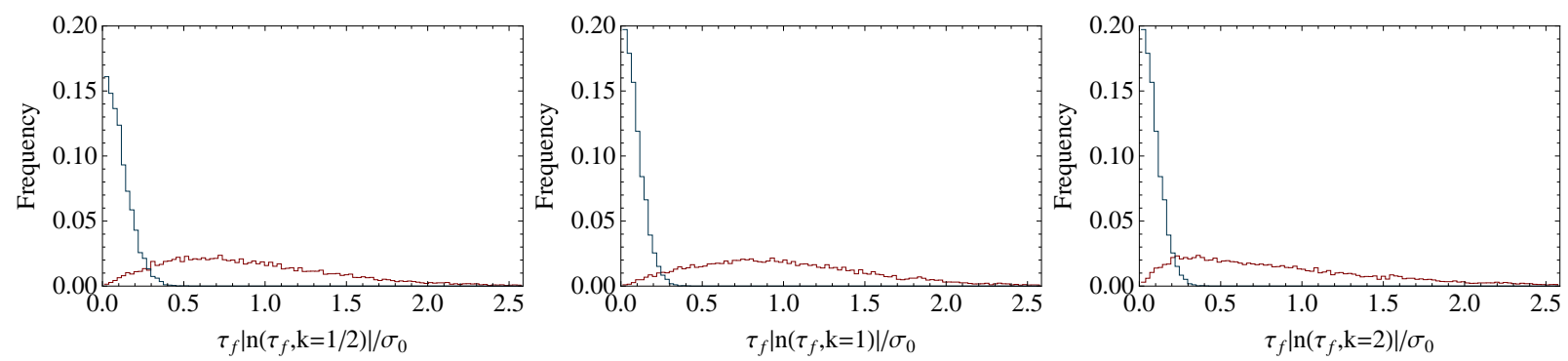

FIG. 5: The distributions of the square root of the power spectrum of eq. (22) in the Fick (blue) and the Kelly (red) theories of diffusion for various $k$ at $\tau_{f}$ corresponding to $\vartheta=2$. The results are from Monte Carlo simulations with 10000 points for each simulation. Initial conditions were chosen from a Gaussian distribution with zero mean and unit variance. All other parameters correspond to the values used to obtain the results in Figures 2 and 3. The frequency histograms are normalized to have unit area.

Our discussion till now has been geared towards identifying the fundamental differences between the Fick and Kelly theories of diffusion. However, the observables that we have discussed may not be the best suited for heavy-ion physics. This is because we have looked at the consequences of starting from a given initial condition. The initial conditions for the fireball change from event to event, and averages over events are unlikely to show overdense or underdense regions. At the LHC particle multiplicities are expected to be high enough that one could build up the profile function $n\left(\tau_{f}, \eta\right)$ at the freezeout epoch, $\tau_{f}$, in a single event. Then by observation of a relatively small number of events, one could distinguish between Kelly and Fick diffusion. However, other techniques are required when the particle multiplicities are smaller.

It would be simplest to construct observables that depend on the power spectrum at freezeout. In an event with $N_{t}$ tracks, one has the charge $q_{j}$ of the $j$-th track and its rapidity $\eta_{j}$. Given these, one may construct the power spectrum of the charge

$$
\bar{P}\left(\tau_{f}, k\right)=\left|\sum_{j=1}^{N_{t}} q_{j} \exp \left(-i k \eta_{j}\right)\right|^{2} .
$$

Since the $q_{j}$ are conserved numbers and not their densities, one has $\bar{P}\left(\tau_{f}, k\right)=\tau_{f}^{2} P\left(\tau_{f}, k\right)$. One may similarly construct the power spectrum of any conserved quantity: $S, B$ or even the proton number, by replacing $q_{j}$ in the above formula by the quantum number under discussion. Again, a plot of $\bar{P}\left(\tau_{f}, k\right)$, averaged over events, as a function of $k$ will smear out many of the effects that we have discussed. Some information can be gained by comparing the results with those obtained from 'mixed events', i.e., when tracks from different events are randomly thrown together into an artificial event with the right multiplicity.

We find that the distinction between Kelly and Fick theories can be observed most directly in the event-to-event distribution of $\tau_{f}\left|n\left(\tau_{f}, k\right)\right| \propto \sqrt{\bar{P}\left(\tau_{f}, k\right)}$. The proportionality constant requires knowledge of the initial volume. However, both the first and second order diffusion equations being linear, the overall normalization of the $n$ and $\nu$ are immaterial. We take advantage of this to work with the dimensionless quantities $\sqrt{\bar{P}\left(\tau_{f}, k\right)}=\tau n(\tau, k) / \sigma_{0}$ and $\tau \nu(\tau, k) / \sigma_{0}$, where the dimensional quantity $\sigma_{0}$ need not be specified except when making an actual connection to models of the initial state. Even so, a prediction of the distribution of $\sqrt{\bar{P}\left(\tau_{f}, k\right)}$ is not possible since the initial distribution is not known. However, starting from the same initial distribution the two theories give rise to completely different distributions at freezeout, and the differences can be investigated.

An example is shown in Figure 5. Here we started with initial conditions drawn from a Gaussian distribution with zero mean and unit variance. Then from eq. (11) it is clear that for Fick diffusion, the distribution of $\sqrt{\bar{P}\left(\tau_{f}, k\right)}$ at freezeout is also a Gaussian, with standard deviation $\exp \left[-\mathcal{D} k^{2}\left(1 / \tau_{0}-1 / \tau_{f}\right)\right]$. Note the strong dependence of the variance on $k$, which is also apparent in Figure 5. The distribution of this quantity in the Kelly theory cannot be derived so easily. We determined it through a Monte Carlo simulation, deriving the distribution at freezeout time by evolving many different samples of initial conditions. The resulting distribution is clearly different. It does not peak at zero, the position of the peak shifts with $k$, and it has a very long tail. These features are intimately connected with the transient amplification phenomenon which has been discussed above. Beyond the range shown in the figures, as $k$ decreases, the frequency of small $\sqrt{\bar{P}}$ increase, the tail shrinks, and the distinction between Fick and Kelly theories 
is lost in the limit of $k \rightarrow 0$. Most importantly, the fact that in all theories the long-time behaviour is dominated by Bjorken attenuation means that the distribution of $\bar{P}\left(\tau_{f}, k\right)$ (or its square root) is independent of $\tau_{f}$. Event-to-event distributions of this kind therefore seem to be the most promising observable distinction between first and second order diffusion.

One of the most widely studied signals of a critical end-point of QCD is the distribution of event-to-event fluctuations of conserved quantities contained within a rapidity acceptance window. The present work, with its removable simplifications, shows that these measures could be influenced, possibly strongly, by the nature of the transport process - whether first or second order. Therefore our understanding of the nature of diffusion needs to be improved before event-to-event fluctuations can be interpreted. We have shown that one way to do this is to study the eventto-event distribution of the power spectrum of the conserved charge (see eq. 22), at $\tau_{f}$ for different $k$ (see Figure 5). There are qualitative differences between the results for the two theories of diffusion.

In summary, here are our main conclusions-

1. The Fourier coefficients, $n(\tau, k)$, and the power spectrum, $P(\tau, k)$, of the number density profile (see eq. 3), obtained by Fourier transforming in the rapidity (which is equal to $\eta$ for boost invariant flow) are of interest in the study of diffusion and hydrodynamics. One can construct number density profiles for any quantum number which is conserved in strong interactions. The obvious ones are $B$ (or $\left.N_{p}\right), Q$, and $S$. A power spectrum can also be constructed from experimental data (see eq. 22), and easily compared to theory.

2. The net conserved number is obviously independent of the kind of hydrodynamics. As a result, the event-to-event distribution of this quantity tells us directly about initial conditions. The net charge within a given rapidity window, however, may evolve with the hydrodynamics (see Figure 4). Since freezeout may occur at different times for different centrality, this may show up as a dependence of the net observed charge on centrality.

3. The Fourier modes give interesting information on the nature of the transport theory. In Kelly (second order) diffusion, the power in short range modes, i.e., modes with large $k$, could be large. This is impossible in Fick (first order) hydrodynamics. The simplest of observables, i.e., a plot of $\bar{P}\left(\tau_{f}, k\right)=\tau_{f}^{2}\left|n\left(\tau_{f}, k\right)\right|^{2}$ as a function of $k$, for an event, can potentially distinguish between these two theories. One thing to note is that this must be done on an event-to-event basis. Averaging over events before constructing the power spectrum could wash out the signal.

4. Event-to-event distributions of the observed power spectrum (see eq. 22), or its square root, for one or more values of $k$ can potentially distinguish between Fick and Kelly theories of diffusion (see Figure 5 and the corresponding discussion in the text).

5. If the Kelly theory is ruled out by such observables, then one can construct an upper bound on the transport coefficient,

$$
\mathcal{D} \leq \tau_{f} \sinh ^{2} \Delta \eta
$$

using the rapidity interval $\Delta \eta$ over which all structure has been washed out and an independent estimate of $\tau_{f}$.

This work was motivated by discussions at the INT program "The QCD Critical Point". SG would like to thank the participants, especially V. Koch, G. Roland, M. Stephanov and N. Xu, for discussions, and gratefully acknowledge the hospitality of the Institute of Nuclear Theory at the University of Washington during this period.

[1] M. A. Stephanov, K. Rajagopal and E. V. Shuryak, Phys. Rev., D 60 (1999) 114028;

M. A. Stephanov, K. Rajagopal and E. Shuryak, Phys. Rev. Lett., 81 (1998) 4816;

M. Asakawa, U. Heinz and B. Muller, Phys. Rev. Lett., 85 (2000) 2072;

S. Jeon and V. Koch, Phys. Rev. Lett., 85 (2000) 2076;

S. Jeon and V. Koch, eprint hep-ph/0304012;

M. A. Stephanov, eprint arXiv:0809.3450.

[2] Y. Hatta and M. A. Stephanov, Phys. Rev. Lett., 91 (2003) 102003.

[3] J. Cleymans and K. Redlich, Phys. Rev. Lett., 81 (1998) 5284.

[4] S. R. de Groot, W. A. van Leeuwen and Ch. G. van Weert, Relativistic Kinetic Theory, North Holland, Amsterdam, 1980; B. Betz, D. Henkel and D. H. Rischke, eprint arxiv:0812.1440.

[5] R. Rapp and H. van Hees, arxiv:0803.0901.

[6] S. Wicks, W. Horowitz, M. Djordjevic and M. Gyulassy, Nucl. Phys., A 784 (2007) 426.

[7] S. Caron-Huot and G. D. Moore, Phys. Rev. Lett., 100 (2008) 052301. 
[8] S. S. Gubser, Phys. Rev., D 74 (2006) 126005;

J. Casalderrey-Solana and D. Teaney, Phys. Rev., D 74 (2006) 085012;

C. P. Herzog et al., J. H. E. P., 07 (2006) 013.

[9] Y. Akamatsu, T. Hatsuda and T. Hirano, arXiv:0809.1499.

[10] G. Wolschin, Prog. Part. Nucl. Phys., 59 (2007) 374.

[11] R. S. Bhalerao and S. Gupta, Phys. Rev., C 77 (2008) 014902.

[12] P. Kovtun and A. Ritz, eprint arXiv:0806.0110;

J. Mas, J. Shock and J. Tarrio, eprint arXiv:0811.1750;

B. Kerbikov, eprint arXiv:0811.3855.

[13] D. C. Kelly, Am. J. Phys., 36 (1968) 585.

[14] S. Gupta, e-print arXiv:0709.2355.

[15] L. N. Trefethen and M. Embree, Spectra and Pseudospectra, Princeton University Press, Cambridge, USA, 2005. 